\title{
Characterization of Unknown Compounds from Stainless Steel Plates in Matrix-Assisted Laser Desorption/Ionization Mass Spectrometry
}

\author{
Hyo-Jik Yang, a,b Kyu Hwan Park, ${ }^{\mathrm{b}}$ Hyun Sik Kim, ${ }^{\mathrm{b}}$ and \\ Jeongkwon Kim ${ }^{\mathrm{a}}$ \\ a Department of Chemistry, Chungnam National University, Daejeon, Republic of Korea \\ ${ }^{\mathrm{b}}$ Korea Basic Science Institute, 804-1 Yangcheong-Ri, Ochang-Myun, Cheongwon-Gun, Chungcheongbuk-Do, \\ Republic of Korea
}

\begin{abstract}
Peaks originating from unknown compounds on stainless steel plates used in matrix-assisted laser desorption/ionization (MALDI) mass spectrometers are observed around $\mathrm{m} / \mathrm{z} 304.3$, 332.3, 360.4, and 388.4 regardless of the matrix and/or solvent, and are even observed with bare plates. These peaks were characterized using three different types of MALDI-MS instrumentation: MALDI-TOF MS, MALDI-TOF/TOF MS, and MALDI-FTMS. The fragmentation data from MALDI-TOF/TOF MS and accurate mass determination by MALDI-FTMS enabled identification of the chemical formulae and structures. The unknown compounds are, in fact, likely benzylalkylmethylammonium salts, as confirmed by closely matching fragmentation patterns with a commercially available benzalkonium chloride. (J Am Soc Mass Spectrom 2010, 21, 2000-2004) (C) 2010 American Society for Mass Spectrometry
\end{abstract}

$\mathrm{M}$ atrix-assisted laser desorption/ionization (MALDI) is one of the most commonly used soft ionization methods in mass spectrometry (MS) [1]. Moreover, the recent development of MALDI imaging and its popularity has generated renewed interest in MALDI techniques [2]. MALDI-MS is a versatile tool for the detection of large proteins as well as many small molecules (e.g., in vitro drugs or metabolites) [3]. However, the ability to analyze small molecules by MALDI-MS is limited by the relative abundance of peaks in the low $\mathrm{m} / \mathrm{z}(<500)$ region derived from common organic matrices [4], which include $\alpha$-cyano-4-hydroxycinnamic acid (CHCA) and 2,5dihydroxybenzoic acid (DHB), or from matrix-derived fragments and adducts $[5,6]$. These peaks make accurate interpretation of MALDI mass spectra in the low mass region extremely difficult. To circumvent this problem, many studies have focused on modifications of current techniques and/or sample preparation, such as surface-assisted laser desorption/ionization [7, 8], the use of surface-modified plates [9], or the identification of new, less interfering matrices, such as ionic liquids $[4,10]$ and carbon nanotubes [11, 12]. Additionally, regarding matrix-derived peaks, several unidentified peaks are commonly observed at $m / z$ 300-400 even in the absence of matrix material [13].

Address reprint requests to Dr. J. Kim, Department of Chemistry, Chungnam National University, 220 Gung-Dong, Yuseong-Gu, Daejeon, 305-764, Republic of Korea, E-mail: jkkim48105@cnu.ac.kr and Dr. H. S. Kim, Korea Basic Science Institute, 804-1 Yangcheong-Ri, Ochang-Myun, CheongwonGun, Chungcheongbuk-Do 363-883, Republic of Korea, E-mail: fticr@ kbsi.re.kr
The current study used three different MALDI mass spectrometers [MALDI-time-of-flight (TOF) MS, MALDITOF/TOF MS, and MALDI-Fourier transform mass spectrometry (FTMS)] to identify the origin of these unknown peaks. The high sensitivity of MALDI-TOF/ TOF MS for fragment ion analyses, combined with the high mass resolution of MALDI-FTMS for elemental analyses, provided structural determination of the compounds responsible for the unknown peaks.

\section{Experimental}

\section{Materials}

Glucose $(\geq 99 \%)$, sucrose $(\geq 99 \%)$, trifluoroacetic acid (TFA, $\geq 98 \%$ ), 2,5-dihydroxybenzoic acid (DHB, 98\%), $\alpha$-cyano-4-hydroxycinnamic acid (CHCA, 99\%), acetonitrile (ACN, HPLC grade), and benzalkonium chloride (catalog number B6295) were purchased from SigmaAldrich (St. Louis, MO, USA). Stainless steel MALDI plates were obtained from Shimadzu Biotech (Kyoto, Japan) and Bruker Daltonics (Bremen, Germany). $\mu$ Focus MALDI plates were obtained from ASTA (Suwon, South Korea).

\section{Sample Preparation}

Matrices were prepared by dissolving $20 \mathrm{mg}$ of each matrix material (DHB or CHCA) in $1 \mathrm{~mL}$ of water/ACN (50:50, vol/vol) containing 0.1\% TFA for MALDI-TOF MS or water/ACN (30:70, vol/vol) containing $0.1 \%$ TFA for MALDI-TOF/TOF MS and MALDI-FTMS. 
Various combinations of DHB, CHCA, distilled water and ACN were applied to the stainless steel MALDI plate and analyzed to determine that the unknown compounds originated from the stainless steel MALDI plates themselves. Samples were also analyzed on an indium-tin oxide (ITO)-coated glass plate to compare the results with those obtained on the stainless steel plate. Commercially available benzalkonium chloride was also deposited on a stainless steel MALDI plate using a general dried-droplet method [2], wherein $1 \mu \mathrm{L}$ of a mixture of $2 \mu \mathrm{L}$ matrix solution (DHB or CHCA) and $2 \mu \mathrm{L}$ of $2.5 \mathrm{mg} / \mathrm{mL}$ analyte in $50 \% \mathrm{ACN}$ aqueous solution was deposited onto the stainless steel plate. The benzalkonium chloride spots were analyzed with an ultrafleXtreme MALDI-TOF/TOF MS.

\section{Mass Spectrometry}

Mass spectra were obtained using a MALDI-TOF MS (Axima CFR, Shimadzu Biotech, 337-nm nitrogen laser), a MALDI-TOF/TOF MS (Ultraflex II MALDI-TOF/TOF MS or ultrafleXtreme MALDI-TOF/TOF MS from Bruker Daltonics, Smartbeam laser), and a MALDIFTMS (15 T Bruker Apex-Qe FT-ICR MS, $355 \mathrm{~nm}$ Nd:YAG laser). For analyses with the MALDI-TOF MS and MALDI-TOF/TOF MS, all shots were accumulated using the reflectron in positive-ion mode. For the MALDI-FTMS, all shots were accumulated in broadband mode with a 4-MB time-domain signal. Three different MALDI plates were used: a stainless steel MALDI plate from Shimadzu Biotech and a $\mu$ Focus MALDI plate from ASTA were used with the Axima CFR MALDI-TOF MS, while a stainless steel MALDI plate from Bruker Daltonics was used with the MALDITOF/TOF MS and MALDI-FTMS. The stainless steel MALDI plates were repeatedly used after comprehensive washing in an ultrasonic bath with methanol for 5 min, followed by washing with distilled water and gentle wiping with Kimwipes (KimberlyClark, Roswell,
GA, USA). In case of the $\mu$ Focus MALDI plate, a brand new plate was used without cleaning the surface to eliminate any potential contamination during washing steps. MS/MS spectra were obtained from the MALDITOF/TOF MS in positive-ion mode, using a laser pulsed at $500 \mathrm{~Hz}$ and averaging 3000 individual spectra. The irradiated laser power was increased by $20 \%$ for MS/MS experiments relative to that used in MS experiments.

Sodiated glucose $\left[\mathrm{C}_{6} \mathrm{H}_{12} \mathrm{O}_{6}+\mathrm{Na}\right]^{+}$at $m / z 203.05$ and sodiated sucrose $\left[\mathrm{C}_{12} \mathrm{H}_{22} \mathrm{O}_{11}+\mathrm{Na}\right]^{+}$at $m / z 365.10$ were used as calibrants in the MALDI-TOF MS, while Peptide Calibration Standard II from Bruker Daltonics (part number 222570) and arginine cluster peaks were used as calibrants in MALDI-TOF/TOF MS and MALDIFTMS experiments. The results of matched molecular ions were obtained from Molecular Weight Calculator (ver. 6.42; Matthew Monroe) and DataAnalysis (ver. 3.4, Bruker Daltonics) software packages. Molecular formulae were determined with DataAnalysis for combinations of $\mathrm{C}, \mathrm{H}, \mathrm{O}, \mathrm{N}$, and $\pm 1 \mathrm{ppm}$ mass tolerance with even electron configurations.

\section{Results and Discussion}

\section{Investigation of Unknown Contaminants on the Stainless Steel MALDI Plate}

Four different peaks of unknown origin were detected in MALDI-TOF MS experiments with stainless steel MALDI plates. Figure 1 shows the MALDI spectrum of the unknown compounds from $\mathrm{m} / \mathrm{z} 300-400$ using a MALDI 15 T FT-ICR MS with DHB matrix on a stainless steel MALDI plate, where two dominant peaks at $\mathrm{m} / \mathrm{z}$ 332.3 and 360.4, and two minor peaks at $\mathrm{m} / \mathrm{z} 304.3$ and 388.4 were observed. The peaks were also observed with CHCA matrix (Supplementary Figure 1S, which can be found in the electronic version of this article). When using ITO-coated glass as the MALDI substrate,

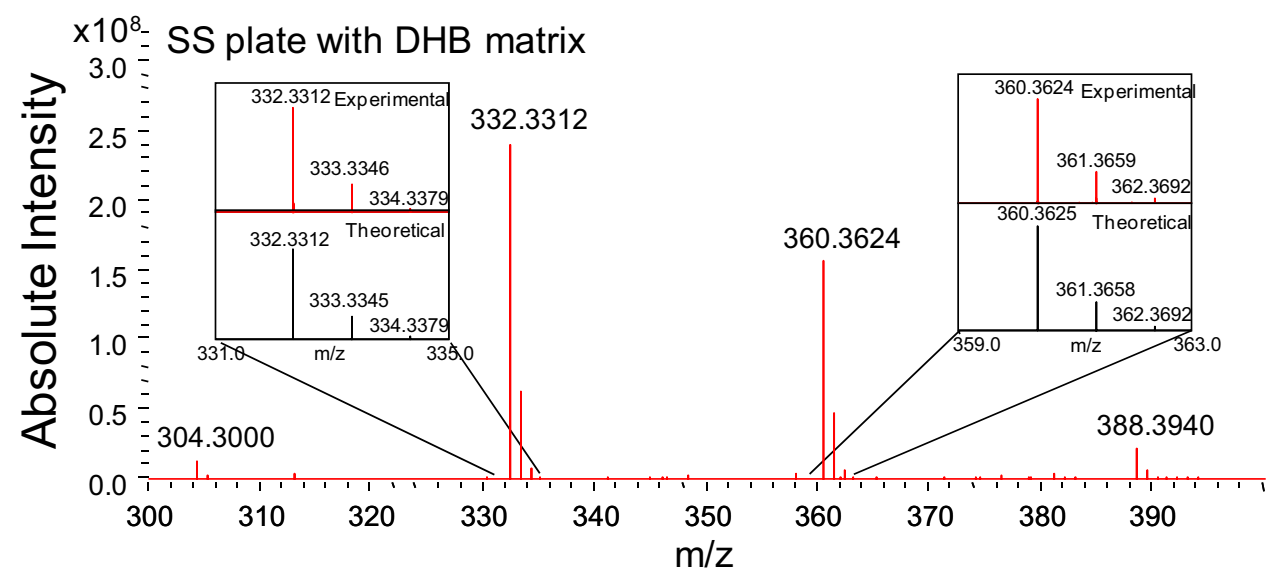

Figure 1. MALDI spectra of the unknown compounds from $m / z$ 300-400, observed using a MALDI 15 T FT-ICR MS on a stainless steel MALDI plate with DHB matrix. The experimental masses were used to find potential candidates for each peak (Table 1). The insets contain the experimental (upper) and the theoretical (lower) mass distributions of the unknown compounds. 
the unknown peaks were not observed (data not shown). Supplementary Figure $2 \mathrm{~S}$ shows that the peaks were also observed with different MALDI plates using (a) MALDI-TOF MS with a DHB matrix solution and with no matrix solution (bare stainless steel plate) and (b) MALDI-TOF/TOF MS with different matrices, including $\mathrm{CHCA}, \mathrm{DHB}, \mathrm{DW}$, and ACN. Laser irradiation of the bare surface of a new, unused $\mu$ Focus MALDI plate also yielded these same peaks (data not shown). Comprehensive washing of the stainless steel MALDI plates in an ultrasonic bath with methanol for $5 \mathrm{~min}$ or in a bath of concentrated nitric acid for $5 \mathrm{~min}$, followed by washing with distilled water could not remove the unknown peaks. These observations strongly demonstrate that the unknown peaks are derived from compounds strongly attached to the surface of the stainless steel MALDI plates.

\section{Determination of Elemental Compositions Using FT-ICR MS}

The $m / z$ values of the unknown peaks in Figure 1 were entered into the Formula Finder algorithm in Molecular Weight Calculator or DataAnalysis. Initially, a mass tolerance of $0.01 \mathrm{Da}$ was used in Molecular Weight Calculator. Elemental composition was limited to combinations of $\mathrm{C}, \mathrm{H}, \mathrm{O}$, and $\mathrm{N}$, along with $\mathrm{Fe}, \mathrm{Cr}$, and $\mathrm{Ni}$ because stainless steel is composed mostly of these metals [14]. However, all of the plausible search results were devoid of any metal. DataAnalysis search results are shown in Table 1. For the peaks at $m / z 332.33118$ and 360.36244 , the theoretical and experimental isotopic mass distributions matched perfectly, as shown in each inset of Figure 1. The exact mass difference between the two adjacent peaks was calculated to be $m / z 28.03126$, equal to the mass of $\mathrm{C}_{2} \mathrm{H}_{4}$, suggesting the existence of a $\mathrm{C}_{2} \mathrm{H}_{4}$ repeating unit in the unknown compounds.

\section{Structural Determination of the Unknown Compounds}

A MALDI-TOF/TOF MS was used to fragment the unknown compounds to determine the structural characteristics. Figure 2a shows the MS/MS spectra of the

Table 1. Identified molecular formulae, based on the mass spectra of MALDI-FTMS. The mass measurement tolerance was set to $\pm 1 \mathrm{ppm}$ and the observed mass values were matched with theoretically calculated mass values using Bruker DataAnalysis. The isotopic distribution of experimental data for each peak perfectly matched that of the theoretical data for each candidate. The experimental and theoretical isotopic distributions for $\mathrm{C}_{23} \mathrm{H}_{42} \mathrm{~N}^{+}$and $\mathrm{C}_{25} \mathrm{H}_{46} \mathrm{~N}^{+}$are shown in the insets of Figure 1

\begin{tabular}{cccrr}
\hline $\begin{array}{c}\text { Observed } \\
\text { mass }\end{array}$ & Candidates & $\begin{array}{c}\text { Theoretical } \\
\text { mass }\end{array}$ & $\begin{array}{c}\text { Error } \\
(\mathrm{mDa})\end{array}$ & $\begin{array}{r}\text { Error } \\
(\mathrm{ppm})\end{array}$ \\
\hline \hline 304.30002 & $\mathrm{C}_{21} \mathrm{H}_{38} \mathrm{~N}^{+}$ & 304.29988 & 0.14 & 0.46 \\
332.33118 & $\mathrm{C}_{23} \mathrm{H}_{42} \mathrm{~N}^{+}$ & 332.33118 & 0.00 & 0.00 \\
360.36244 & $\mathrm{C}_{25} \mathrm{H}_{46} \mathrm{~N}^{+}$ & 360.36248 & -0.04 & -0.11 \\
388.39401 & $\mathrm{C}_{27} \mathrm{H}_{50} \mathrm{~N}^{+}$ & 388.39378 & 0.23 & 0.59 \\
\hline
\end{tabular}

unknown compounds with DHB matrices on a stainless steel MALDI plate. The fragmentation of the unknown compounds was performed at $\mathrm{m} / \mathrm{z} 304.3,332.3,360.4$, and 388.4 (from top to bottom). The fragmentation of peaks at $\mathrm{m} / \mathrm{z} 304.3$ and 388.4 was performed using an Ultraflex II MALDI-TOF/TOF MS, while the fragmentation of peaks at $\mathrm{m} / \mathrm{z} 332.3$ and 360.4 was performed using an ultrafleXtreme MALDI-TOF/TOF MS. The parent ions at $m / z 304.3,332.3,360.4$, and 388.4 differed only by the length of an alkyl chain showed similar structural features, such as an iminium ion $\left[\left(\mathrm{CH}_{3}\right) \mathrm{N}^{+}=\right.$ $\mathrm{CH}_{2}$ ] at $\mathrm{m} / \mathrm{z} 57.8$ and a tropylium ion $\left(\mathrm{C}_{7} \mathrm{H}_{7}{ }^{+}\right)$at $\mathrm{m} / \mathrm{z}$ 90.8. Additionally, all of the molecular ions of the unknown compounds showed a neutral loss of toluene $\left(\mathrm{C}_{6} \mathrm{H}_{5} \mathrm{CH}_{3}, 92.1 \mathrm{Da}\right)$. The loss of an alkyl chain yielded the peak at $m / z 135.9$, which was only observed from the parent ions at $\mathrm{m} / \mathrm{z} 304$ and 332 .

The MALDI MS/MS experimental data is consistent with data obtained from field desorption-tandem mass spectrometry [15] or collision-induced dissociation [1622] of benzylalkyldimethylammonium chloride, also known as benzalkonium chloride (BAC), a quaternary ammonium salt, commonly used as a disinfectant or a preservative [23]. The potential structures obtained from Figure 2a correspond to the structure of the benzalkonium ion. Depending on the length of the alkyl side chain, $B A C$ homologs are named as $C_{12}$-BAC, $\mathrm{C}_{14}-\mathrm{BAC}, \mathrm{C}_{16}-\mathrm{BAC}$, or $\mathrm{C}_{18}$-BAC. The fragmentation of BAC has been studied using tandem MS [15-22], and generates an iminium ion around $m / z 58[15,16]$, a tropylium ion around $m / z$ 91 [15-22], and ions around $\mathrm{m} / \mathrm{z} 136$ from the loss of the alkyl chain $[15,20,22]$. Additionally, a neutral loss of toluene is observed for all of the BAC homolog ions [15-22].

To improve confidence in this identification, a commercial BAC was analyzed by a MALDI TOF/TOF MS. Figure $2 b$ shows the MALDI MS and MALDI MS/MS spectra of a commercial BAC. The commercial BAC provided only two distinct peaks at $\mathrm{m} / \mathrm{z} 304.30\left(\mathrm{C}_{12^{-}}\right.$ BAC) and $m / z 332.33\left(\mathrm{C}_{14}\right.$-BAC). The MS/MS spectra of these two peaks yielded fragmentation patterns similar to those of the unknown compounds originating from the stainless steel MALDI plates (Figure 2a).

\section{Origin of the Unknown Compounds}

The experimental data suggested that the unknown peaks observed in mass spectra acquired from samples on stainless steel MALDI plates were derived from BAC. BACs are commonly used as disinfectants, wetting agents, frothing agents, anti-corrosion agents, and waterproofing materials, and are extensively used in biomedical equipment and pharmaceutical applications [24]. Additionally, quaternary ammonium salts have been used as phase-transfer catalysts [25]. It is reasonable to assume, given its versatility, that BAC was used in the manufacturing of the stainless steel MALDI 

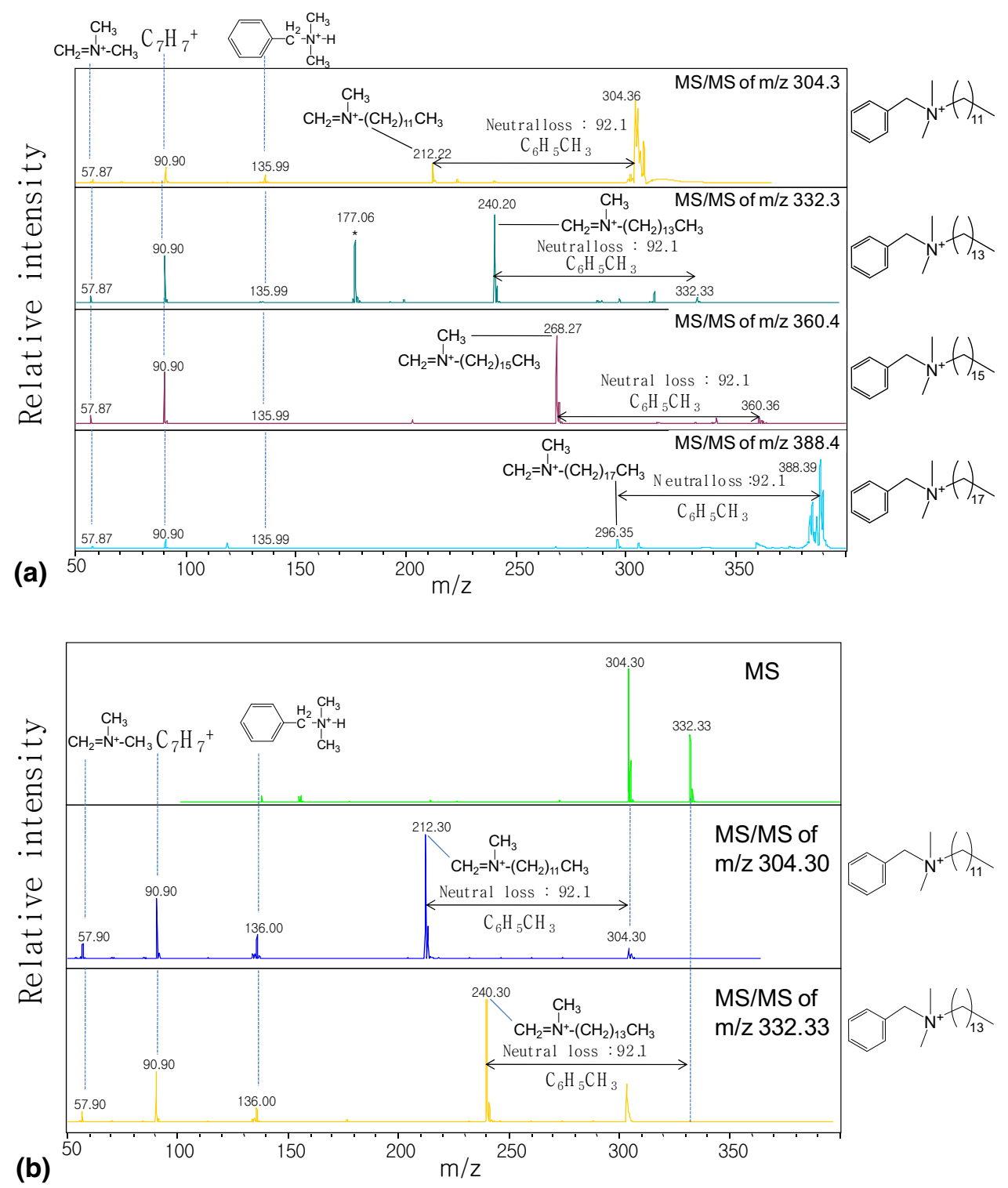

Figure 2. MALDI-TOF/TOF MS spectra with DHB matrices of (a) the unknown compounds acquired on a stainless steel MALDI plate and (b) commercial benzalkonium chloride on ITO-coated glass. (a) A neutral loss of toluene $\left(\mathrm{C}_{6} \mathrm{H}_{5} \mathrm{CH}_{3}, 92.1 \mathrm{Da}\right)$, an iminium ion $\left[\left(\mathrm{CH}_{3}\right) \mathrm{N}^{+}=\mathrm{CH}_{2}\right]$ at $\mathrm{m} / z$ 57.8, and a tropylium ion $\left(\mathrm{C}_{7} \mathrm{H}_{7}{ }^{+}\right)$at $\mathrm{m} / \mathrm{z} 90.8$ were observed in all MS/MS spectra of the unknown compounds. The peak at $\mathrm{m} / \mathrm{z} 177.06$ in the MS/MS spectrum of $\mathrm{m} / \mathrm{z} 332.3$ with DHB matrix was believed to be from an impurity, $[\mathrm{DHB}+\mathrm{Na}]^{+}$. The potential structure of each parent ion is drawn in the right side of each MS/MS spectrum. (b) The fragmentation of commercial benzalkonium chloride was performed at $\mathrm{m} / \mathrm{z} 304.30$ and 332.33 using an ultrafleXtreme MALDI-TOF/TOF MS. A neutral loss of a toluene molecule $\left(\mathrm{C}_{6} \mathrm{H}_{5} \mathrm{CH}_{3}, 92.1 \mathrm{Da}\right)$, an iminium ion $\left[\left(\mathrm{CH}_{3}\right) \mathrm{N}^{+}=\mathrm{CH}_{2}\right]$ at $\mathrm{m} / z$ 57.90, and a tropylium ion $\left(\mathrm{C}_{7} \mathrm{H}_{7}{ }^{+}\right)$at $\mathrm{m} / z 90.90$ were observed in all MS/MS spectra. A peak at $\mathrm{m} / z 136.00$ generated from the loss of the alkyl chain was also observed. The potential structure of each parent ion is also drawn in the right side of each MS/MS spectrum.

plates, perhaps as an anti-corrosion agent or as a disinfectant.

\section{Conclusions}

Various MALDI-MS experiments were used to determine the structural details of compounds that gave rise to unknown peaks in mass spectra acquired on stainless steel MALDI plates. The results provided here provide evidence that the unknown compounds are BACs and originate from the stainless steel MALDI plates themselves, not from the MALDI matrix solutions. The high mass accuracy of a MALDI 15 T FT-ICR MS allowed precise formula identification and provided partial structural information. Further structural information was provided by MS/MS data from a MALDI-TOF/ TOF instrument. The identification of these previously unknown compounds is important for the analysis of 
small molecules by MALDI-MS because the assigned peaks can be used as internal calibrants.

\section{Acknowledgments}

The author appreciate helpful discussions of Dr. Yangsun Kim. The authors acknowledge support for this work by the Korea Research Foundation Grant funded by the Korean Government2008-331-C00188 to J.K. and by KBSI grant G30122 to H.S.K. Portions of this research (Molecular Weight Calculator) were supported by the W. R. Wiley Environmental Molecular Science Laboratory (a national scientific user facility sponsored by the U.S. Department of Energy's Office of Biological and Environmental Research and located at PNNL). PNNL is operated by Battelle Memorial Institute for the U.S. Department of Energy under contract DE-AC05-76RL0 1830.

\section{Appendix A Supplementary Material}

Supplementary material associated with this article may be found in the online version at doi:10.1016/ j.jasms.2010.08.010.

\section{References}

1. Hillenkamp, F.; Karas, M.; Beavis, R. C.; Chait, B. T. Matrix-Assisted Laser Desorption/Ionization Mass Spectrometry of Biopolymers. Anal. Chem. 1991, 63, 1193A-1203A.

2. Wang, C. H.; Li, J.; Yao, S. J.; Guo, Y. L.; Xia, X. H. High-Sensitivity Matrix-Assisted Laser Desorption/Ionization Fourier Transform Mass Spectrometry Analyses of Small Carbohydrates and Amino Acids Using Oxidized Carbon Nanotubes Prepared by Chemical Vapor Deposition as Matrix. Anal. Chim. Acta 2007, 604, 158-164.

3. Hsieh, Y.; Chen, J.; Korfmacher, W. A. Mapping Pharmaceuticals in Tissues using MALDI Imaging Mass Spectrometry. J. Pharmacol. Toxicol. Methods 2007, 55, 193-200.

4. Tholey, A.; Heinzle, E. Ionic (Liquid) Matrices for Matrix-Assisted Laser Desorption/Ionization Mass Spectrometry-Applications and Perspectives. Anal. Bioanal. Chem. 2006, 386, 24-37.

5. Hao, C.; Ma, X.; Fang, S.; Liu, Z.; Liu, S.; Song, F.; Liu, J. Positive- and Negative-Ion Matrix-Assisted Laser Desorption/Ionization Mass Spectrometry of Saccharides. Rapid Commun. Mass Spectrom. 1998, 12, 345trom 348 .

6. Harris, W. A.; Janecki, D. J.; Reilly, J. P. Use of Matrix Clusters and Trypsin Autolysis Fragments as Mass Calibrants in Matrix-Assisted Laser Desorption/Ionization Time-of-Flight Mass Spectrometry. Rapid Commun. Mass Spectrom. 2002, 16, 1714-1722.

7. Liu, Q.; Xiao, Y.; Pagan-Miranda, C.; Chiu, Y. M.; He, L. Metabolite Imaging Using Matrix-Enhanced Surface-Assisted Laser Desorption/ Ionization Mass Spectrometry (ME-SALDI-MS). J. Am. Soc. Mass Spectrom. 2009, 20, 80-88.

8. Watanabe, T.; Kawasaki, H.; Yonezawa, T.; Arakawa, R. SurfaceAssisted Laser Desorption/Ionization Mass Spectrometry (SALDI-MS) of Low Molecular Weight Organic Compounds and Synthetic Polymers Using Zinc Oxide ( $\mathrm{ZnO})$ Nanoparticles. J. Mass Spectrom. 2008, 43, 1063-1071.

9. Wei, J.; Buriak, J. M.; Siuzdak, G. Desorption-Ionization Mass Spectrometry on Porous Silicon. Nature 1999, 399, 243-246.

10. Tholey, A.; Zabet-Moghaddam, M.; Heinzle, E. Quantification of Peptides for the Monitoring of Protease-Catalyzed Reactions by MatrixAssisted Laser Desorption/Ionization Mass Spectrometry Using Ionic Liquid Matrixes. Anal. Chem. 2006, 78, 291-297.

11. Pan, C.; Xu, S.; Hu, L.; Su, X.; Ou, J.; Zou, H.; Guo, Z.; Zhang, Y.; Guo, B. Using Oxidized Carbon Nanotubes as Matrix for Analysis of Small Molecules by MALDI-TOF MS. J. Am. Soc. Mass Spectrom. 2005, 16, 883-892.

12. Ren, S. F.; Guo, Y. L. Oxidized Carbon Nanotubes as Matrix for Matrix-Assisted Laser Desorption/Ionization Time-of-Flight Mass Spectrometric Analysis of Biomolecules. Rapid Commun. Mass Spectrom. 2005, 19, 255-260.

13. Yang, H. J.; Lee, A.; Lee, M. K.; Kim, W.; Kim, J. Detection of Small Neutral Carbohydrates Using Various Supporting Materials in Laser Desorption/Ionization Mass Spectrometry. Bull. Korean Chem. Soc. 2010, 31, 35- 40.

14. Kumar, R.; Srivastava, P. K.; Srivastava, S. P. Leaching of Heavy Metals (Cr, Fe, and Ni) from Stainless Steel Utensils in Food Simulants and Food Materials. Bull. Environ. Contam. Toxicol. 1994, 53, 259-266.

15. Jackson, A. T.; Jennings, R. C. K.; Scrivens, J. H.; Green, M. R.; Bateman, R. H. The Characterization of Complex Mixtures by Field Desorption Tandem Mass Spectrometry. Rapid Commun. Mass Spectrom. 1998, 12, 1914-1924.

16. Takeoka, G. R.; Dao, L. T.; Wong, R. Y.; Harden, L. A. Identification of Benzalkonium Chloride in Commercial Grapefruit Seed Extracts. J. Agr. Food. Chem. 2005, 53, 7630-7636.

17. Ferrer, I.; Furlong, E. T. Accelerated Solvent Extraction Followed by On-Line Solid-Phase Extraction Coupled to Ion Trap LC/MS/MS for Analysis of Benzalkonium Chlorides in Sediment Samples. Anal. Chem. 2002, 74, 1275-1280.

18. Para, B. V.; Nunez, O.; Moyano, E.; Galceran, M. T. Analysis of Benzalkonium Chloride by Capillary Electrophoresis-Tandem Mass Spectrometry. Electrophoresis 2006, 27, 2225-2232.

19. Nunez, O.; Moyano, E.; Galceran, M. T. Determination of Quaternary Ammonium Biocides by Liquid Chromatography-Mass Spectrometry. J. Chromatogr. A 2004, 1058, 89-95.

20. Heike, S. T.; Rainer, T.; Sebastian, B.; Ernst, A. S.; Klaus, K. M. Fate of Benzalkonium Chloride in a Sewage Sludge Low Temperature Conversion Process Investigated by LC-LC/ESI-MS/MS. CLEAN-Soil Air Water 2007, 35, 81-87.

21. Ferrer, I.; Furlong, E. T. Identification of Alkyl Dimethylbenzylammonium Surfactants in Water Samples by Solid-Phase Extraction Followed by Ion Trap LC/MS and LC/MS/MS. Environ. Sci. Technol. 2001, 35, 2583-2588.

22. Merino, F.; Rubio, S.; Perez-Bendito, D. Mixed Aggregate-Based AcidInduced Cloud-Point Extraction and Ion-Trap Liquid ChromatographyMass Spectrometry for the Determination of Cationic Surfactants in Sewage Sludge. J. Chromatogr. A 2003, 998, 143-154.

23. Purohit, A.; Kopferschmitt-Kubler, M. C.; Moreau, C.; Popin, E.; Blaumeiser, M.; Pauli, G. Quaternary Ammonium Compounds and Occupational Asthma. Int. Arch. Occup. Environ. Health 2000, 73, 423427.

24. Patrauchan, M. A.; Oriel, P. J. Degradation of Benzyldimethylalkylammonium Chloride by Aeromonas hydrophila sp K. J. Appl. Microbiol. 2003, 94, 266-272.

25. Ohtani, N.; Morimoto, Y.; Naitou, M.; Kasuga, Y.; Tsuchimoto, D. Phenomenological Properties and Phase Behavior of Benzylalkyldimethylammonium Salts in the Presence of Benzene and Electrolyte Solutions. Langmuir 2001, 17, 3829-3835. 